\title{
Application of an aviation model of incident reporting and investigation to the neurosurgical scenario: method and preliminary data
}

\author{
Paolo Ferroli, M.D., ${ }^{1}$ Dario Caldiroli, M.D., ${ }^{1}$ Francesco Acerbi, M.D., Ph.D., ${ }^{1}$ \\ Maurizio Scholtze, M.A., ${ }^{2}$ Alfonso Piro, ${ }^{2}$ Marco Schiariti, M.D., 1 \\ Eleonora F. Orena, Ph.D., ${ }^{1}$ Melina Castiglione, M.D., 1 Morgan Broggi, M.D., 1 \\ Alessandro Perin, M.D., Ph.D., ${ }^{1}$ and Francesco DiMeco, M.D. ${ }^{1}$ \\ ${ }^{1}$ Department of Neurosurgery, Fondazione Istituto Neurologico “Carlo Besta," Milano; and 2Aviationlab, Roma, \\ Italy
}

Object. Incident reporting systems are universally recognized as important tools for quality improvement in all complex adaptive systems, including the operating room. Nevertheless, introducing a safety culture among neurosurgeons is a slow process, and few studies are available in the literature regarding the implementation of an incident reporting system within a neurosurgical department. The authors describe the institution of an aviation model of incident reporting and investigation in neurosurgery, focusing on the method they have used and presenting some preliminary results.

Methods. In 2010, the Inpatient Safety On-Board project was developed through cooperation between a team of human factor and safety specialists with aviation backgrounds (DgSky team) and the general manager of the Fondazione Istituto Neurologico Carlo Besta. In 2011, after specific training in safety culture, the authors implemented an aviation-derived prototype of incident reporting within the Department of Neurosurgery. They then developed an experimental protocol to track, analyze, and categorize any near misses that happened in the operating room. This project officially started in January 2012, when a dedicated team of assessors was established. All members of the neurosurgical department were asked to report near misses on a voluntary, confidential, and protected form (Patient Incident Reporting System form, Besta Safety Management Programme). Reports were entered into an online database and analyzed by a dedicated team of assessors with the help of a facilitator, and an aviation-derived root cause analysis was performed.

Results. Since January 2012, 14 near misses were analyzed and classified. The near-miss contributing factors were mainly related to human factors (9 of 14 cases), technology ( 1 of 14 cases), organizational factors ( 3 of 14 cases), or procedural factors ( 1 of 14 cases).

Conclusions. Implementing an incident reporting system is quite demanding; the process should involve all of the people who work within the environment under study. Persistence and strong commitment are required to enact the culture change essential in shifting from a paradigm of infallible operators to the philosophy of errare humanum est. For this paradigm shift to be successful, contributions from aviation and human factor experts are critical.

(http://thejns.org/doi/abs/10.3171/2012.9.FOCUS12252)

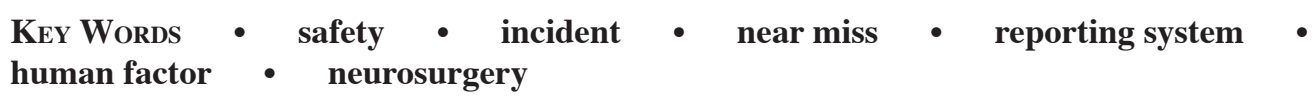

$\mathrm{M}$ EDICAL mistakes are traditionally poorly accepted; when they are recognized, it is generally for punitive purposes. This pattern was also true in the airline industry until the Dryden accident in 1989, the disaster that paved the way for a thorough review of both the educational and evaluative processes of aircraft pilots. This review led to a dramatic improvement in aviation safety, when experts understood for the first time the role that human factors play in determining the final outcome of errors.

In recent years, health care organizations have increasingly focused their attention on safety issues. Sev-

\footnotetext{
Abbreviations used in this paper: $\mathrm{EU}=$ European Union; ISOB = Inpatient Safety On-Board; PIRS = Patient Incident Reporting System.
}

eral national agencies have committed themselves to improving safety standards within their national health care systems (Italian Ministry of Health documentation: Sentinel Events Monitoring Protocol [July 2009]; Safety Manual in the Operation Room [July 2009]; Methods and Analysis for Managing Health Care Risks-Root Cause Analysis [September 2009]; Risk Management in Medicine-Errors Problem [March 2004]), and the patient safety approach presents strong similarities with risk management protocols in the aeronautic safety management system. ${ }^{16}$

Incident precursors may not be solely related to technical or nontechnical skills of the operators; therefore, their analysis should include the complex framework in which all organizational or institutional components are directly or indirectly evaluated. ${ }^{23}$ In the incident investi- 


\section{P. Ferroli et al.}

gation process "latent failures" are normally referred to as "organizational layers"; therefore, it is very important to determine the relative contribution of frontline operators' honest mistakes (active failures) as compared with organizational errors.

The experience gained in the aviation safety system has demonstrated that an accident, the equivalent of a medical "sentinel event" that causes serious patient harm or death, is usually preceded by many weak signals, for example, near misses or errors that might have led to an adverse event but were fortuitously intercepted or did not cause any harmful consequences. These sequences of events are not always completely clear and straightforward; more importantly, they are not always promptly detected and analyzed so that they can be prevented in the future. ${ }^{14}$ Incident reporting systems can thus be important tools for quality improvement in complex adaptive systems, including the operating room. ${ }^{21}$ We describe the application of an aviation model of incident reporting and investigation to the neurosurgical scenario, providing an outline of the method we used as well as some preliminary results.

\section{Methods}

\section{The Start-Up}

In 2010, the ISOB project was developed through cooperation between a team of human factor and safety specialists with aviation backgrounds (DgSky team) and the general manager of the Fondazione Istituto Neurologico Carlo Besta. In 2011, after specific training in safety culture, we implemented an aviation-derived prototype of incident reporting within the Department of Neurosurgery. We then developed an experimental protocol to track, analyze, and categorize any near misses that happened in the operating room. This project officially started in January 2012, when a dedicated team of assessors was established.

\section{Education and Recruitment of Reporting Staff}

All of the people working within the operating room of our neurosurgical department were taught key issues in safety culture and human factors through dedicated crew resource management classes led by facilitators with aviation backgrounds. The crew resource management classes emphasized the importance of communication, teamwork, situation awareness, stress management, and decision making in relation to the working environment. The impact of incident reporting systems in aviation was also emphasized to highlight the role of human factors in all complex adaptive systems, including the operating room. Our aim in this project was to create an incident reporting database repository to monitor performance and trend analysis.

To ensure reporting compliance and protect data and staff members, the incident reporting system was launched under a confidential, voluntary model, following the "just culture" principles in accordance with our institution safety policy guidelines. ("Just culture" is defined as "a culture in which frontline operators or others are not punished for actions, omissions, or decisions taken by them that are commensurate with their experience and training, but in which gross negligence, willful violations, and destructive acts are not tolerated." International Civil Aviation Organisation 2007.) The PIRS (Besta Safety Management Programme) was designed and repeatedly shown and explained to all staff members as an ad hoc, voluntary, confidential, protected, anonymous reporting form (Fig. 1). This form was made available online on our institute Intranet. To avoid any lexical confusion or misunderstandings, we reinforced the definition and classification of near misses (our main object of the project) to all staff members and instructed and encouraged all employees - in particular, senior staff members-to report near misses and incidents.

\section{Data Collection}

Completed PIRS forms were only accessible by the coordinator of the team of assessors, and he (D.C.) was the sole person to process and transfer the forms to an Intranet-based reporting system. Assessors, bound by a confidentiality policy that protected both staff members and the content of the PIRS forms, evaluated the data.

\section{The PIRS Form}

We designed the data collection form specifically for reporting incidents within a neurosurgical operating room (Fig. 1). The resulting PIRS form is a very simple double-sided sheet that combines previously defined international best practices in health care incident reporting with expert recommendations from our aviation partners. Questions (some in multiple-choice form) about specific risk factors were inserted: When and Where (location and phase of surgery), What (description of the context and factors' sequence), How (how the incident was detected, and what remedies were put in place), Impact (self-perceived severity and self-estimation of the potential consequences/effects along with their estimated duration), Potential for recurrence (reasons for possible recurrence of the incident), and Suggestions (suggestions to improve safety measures and to prevent similar incidents from happening again). Special attention was paid to causal and contributory factors related to any human, technological, procedural, or organizational factors. The PIRS form was also designed to help the team of assessors by taking into account the reporters' feelings and suggestions.

\section{Understanding Errors, Data Analysis, and Assessment and} Countermeasures

A dedicated team of assessors led by the team coordinator and assisted by a facilitator, both with aviation backgrounds, met monthly to analyze any near-miss reports. Facilitation was tailored to reproduce a structured aviationbased root cause analysis brainstorming session. We decided to perform a retrospective analysis of every single event, starting from detecting the incident effects and then going backward, looking for any causal and contributing factors. This reverse process continued until the most remote, deep factors underlying that incident were postulated. Data clustering followed, and corrective action recommendations and proposals were made. The assessment team then used these interpretations to create a cause-effect flowchart. 


\section{Development of an incident reporting system}

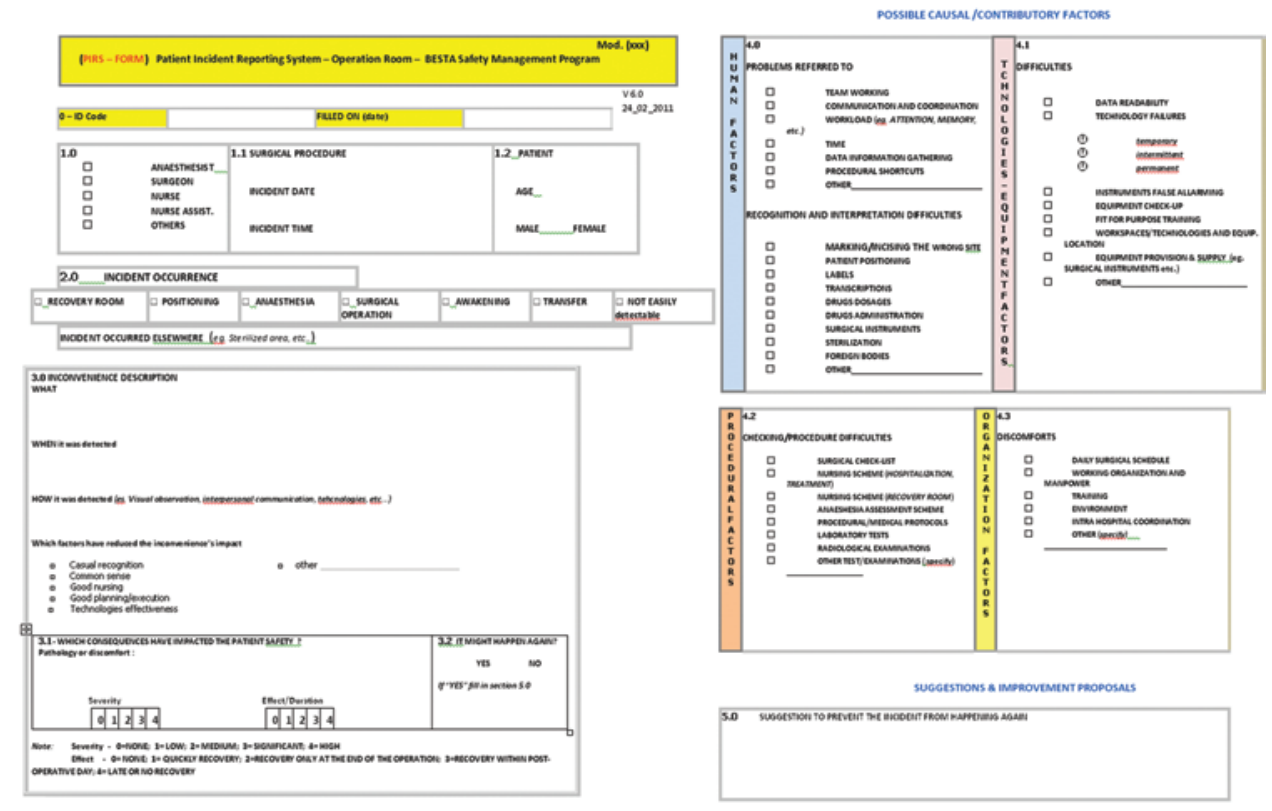

FIG. 1. The PIRS form, a specifically designed double-sided incident reporting form for neurosurgery near-miss events.

Reported near misses were classified and rated by event severity and duration, and the severity score was double-checked by the team of assessors. An additional score subdivided events into 4 areas: human, technological, organizational, and procedural factors, which further defined the risk level. Those areas were further defined on subsequent versions of the PIRS form to help reporters and assessors better identify any weakness in the system. For instance, for any reported event, we investigated how such incidents were related to human, organizational, or behavioral problems (for example, lack of communication, teamwork, decision making, or leadership; or influence of time pressure, workload, misinterpretation, high/ low expectation, multitasking activities, and so forth); technological issues (for example, fit-to-purpose technologies, training, performance standardization on humanmachine interface issues, warnings-alarm management, spatial confusion, equipment setup and maintenance, and so forth); and procedures and protocols consistency (for example, checklist effectiveness, drug dosing, task allocations, and so forth). Errors and trigger events were rated and grouped, starting from the most frequent or severe errors down to the least severe. The adopted countermeasures were those suggested on the reporting forms, solutions found in the literature, or consensus strategies that came from the sessions.

\section{Near-Miss Risk Stratification}

Near-miss risk stratification resulted from the PIRS form analysis process described above during the periodic meetings of the team of assessors. Risk was stratified based on score evaluation and through 2-phase meetings led by the team coordinator (Fig. 2).

Phase 1. At the end of the incident analysis, the team of assessors was asked to score the consequences of the event in terms of their severity (none [0], low [1], medium
[2], significant [3], high [4]) and effect duration (none [0], quick recovery [1], recovery only at the end of the operation [2], recovery within postoperative Day 1 [3], late or no recovery [4]). The total score (severity + duration) ranged from 0 to 8 .

Phase 2. The team of assessors was also asked to assess and score the impact of the 4 systemic components (human factors, technology, organizational factors, and procedural factors). The maximum score that could be attributed to the systemic components was 8 , and the team of assessors was allowed to score each single factor, assessing its relative importance in the context of that specific event. The sum of both the severity/duration score and the score of the impact of every systemic component was assumed to give an idea of the event-related risk and was arbitrarily stratified in 5 risk areas. An example of a near-miss final report is featured in Fig. 2.

This process allowed us to analyze the real relevance of the incident, keeping all of the components that were possibly involved in the account. Five levels of risk subcategories were introduced to generate a system that might provide a correlation between the level of risk and the need for countermeasures. In addition, wide and flexible categories allowed future fine-tuning of trend analysis within the same subcategories-for example, how much the classified risk is going toward the area of major riskand therefore identifying an alert zone.

We decided to insert a specific field into the PIRS form for the reporter to describe his or her own risk perception to draw parallels with the results from the assessment team.

\section{Results}

The analysis results for the 14 cases we collected between January and May 2012 are illustrated in Table 1. Four areas of interest concerning both the system and the 


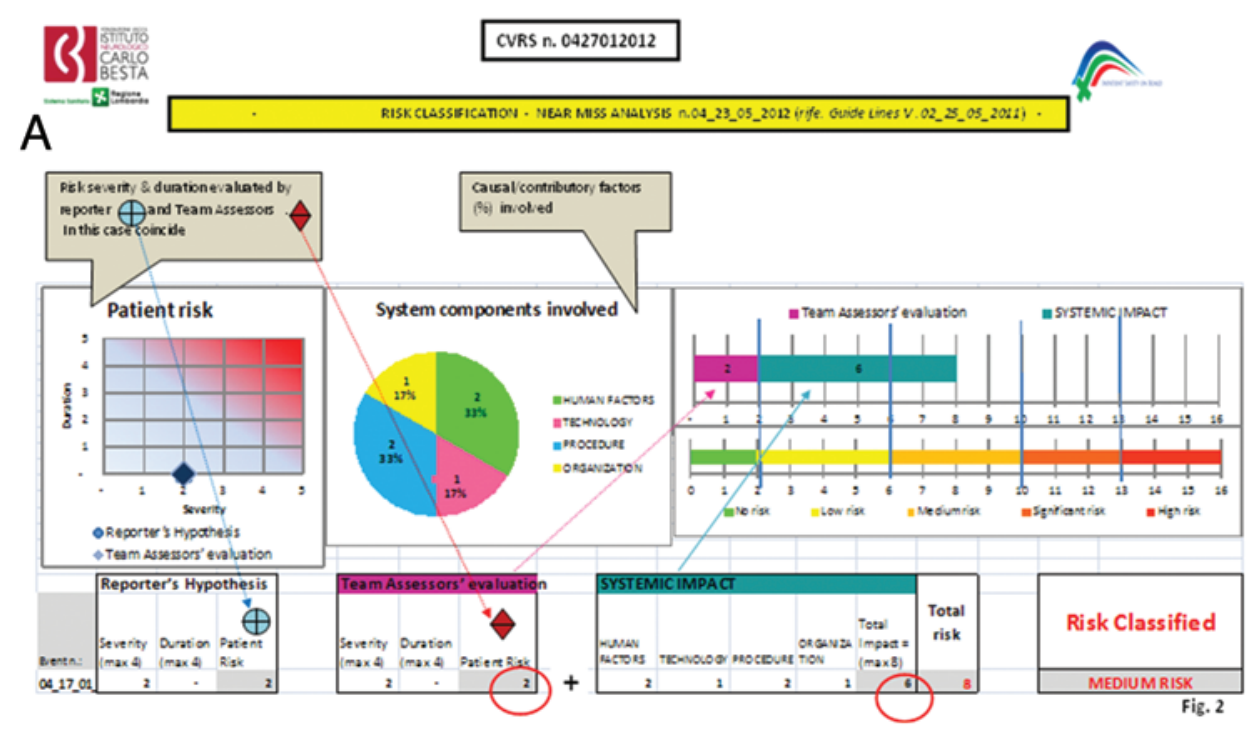

TAXONOMY
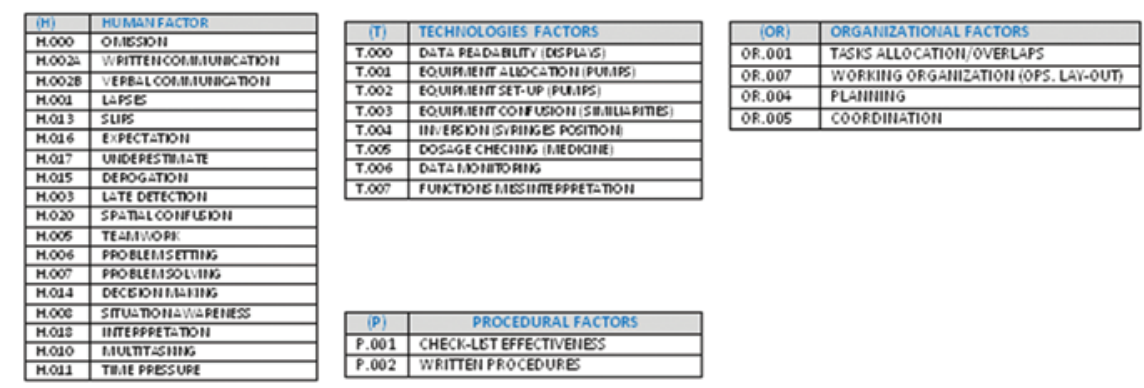
T.03

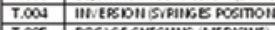

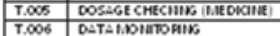

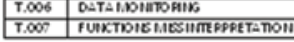

B

REMARKS - REMEDIAL ACTIONS \& MONITORING - NEAR MISS n.04_23_05_2012

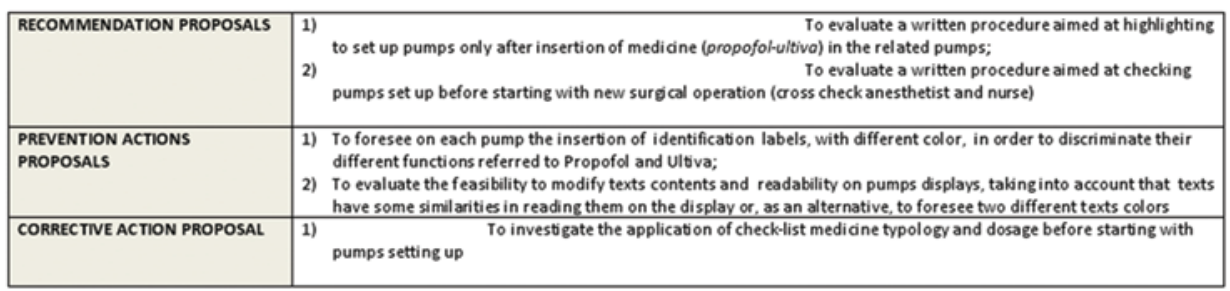

\begin{tabular}{|l|l|}
\hline FEEDBACK TO REPOURTER & DURING THE MEETING \\
\hline
\end{tabular}

MONITORING (ee Repetiviveness of analogues events including related errors typologes; a dions under aken effectiveness, etc)

Within 4 months from the event - accountable persons: Team Assessor Coordinator (TAC) in coordination with Risk manager (RM)

Within 8 months from the event - accountable persons: Team Assessor Coordinator (TAC) in coordination with Risk manager (RM)

Fig. 2. Example of a near-miss final report after evaluation by the team of assessors. A: The incident reporting dashboard summarizes the analysis performed by the team of assessors for a case of prolonged arterial hypotension due to a mistake in the drug pump setting. As far as risk stratification is concerned, this event fell into the middle risk zone, with a team score of 8 (event effect + human factor + technology + organizational factor + procedure $=2+2+1+1+2)$. B: Preventing actions and monitoring activities are also shown.

components can be identified: human factors, technology involved, organizational factors, and procedures. These areas were analyzed and visualized as a flowchart to help both the reporters and the team of assessors identify any weaknesses or issues within any reported event. The following contributing factors were found to recur in all of the 14 near misses we analyzed: 1) human factor: 9 of 14 cases - in particular, omission, lack of communication, late detection, lack of teamwork, problem setting, problem solving, situation awareness, multitasking, and time pressure; 2) technologies: 1 of 14 cases - in particular, problems with equipment allocation, instrument setup, interface similarities, and data interpretation; 3) organizational factors: 3 of 14 cases -in particular, task allocation/overlap, working 


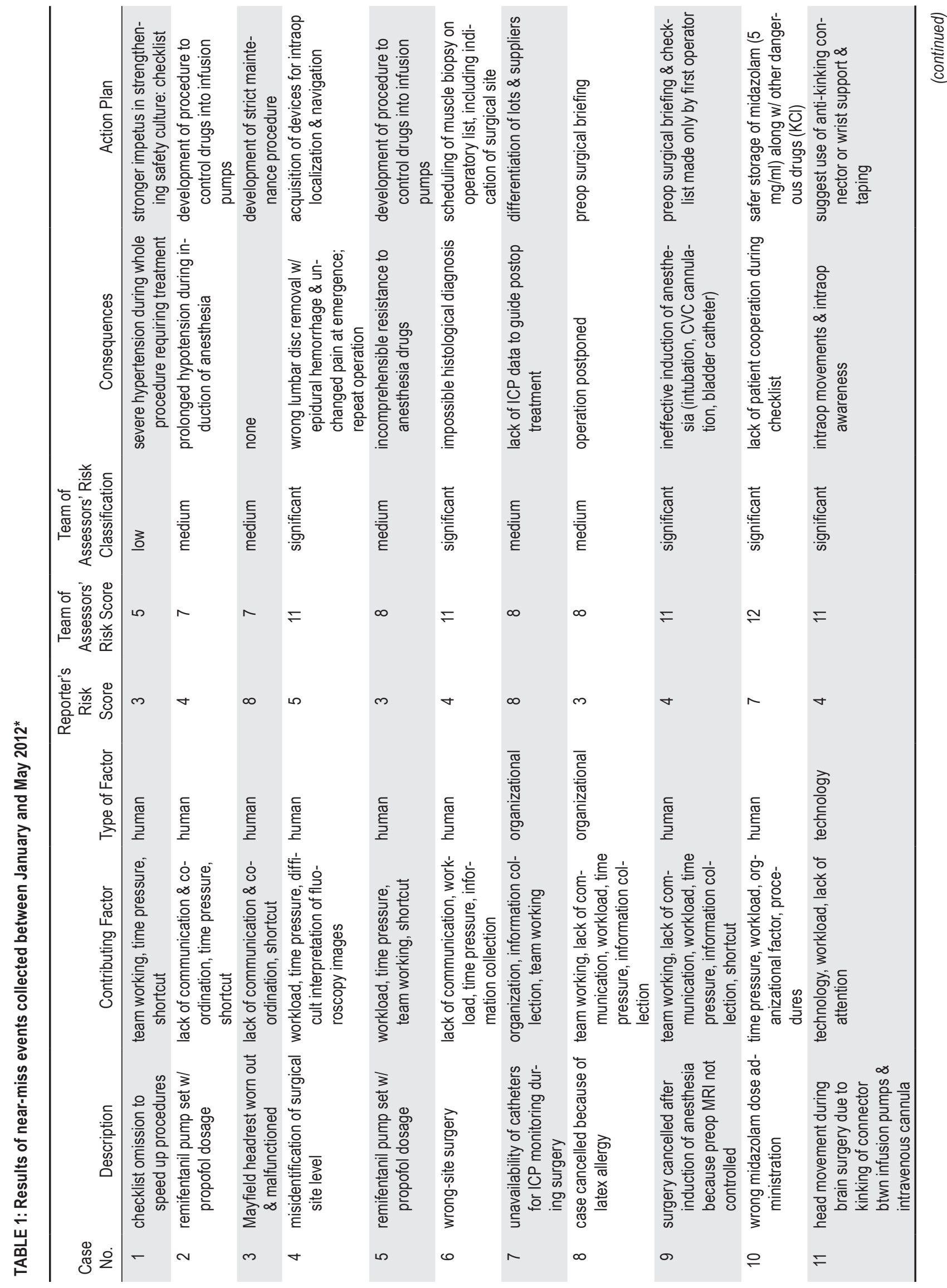




\section{P. Ferroli et al.}

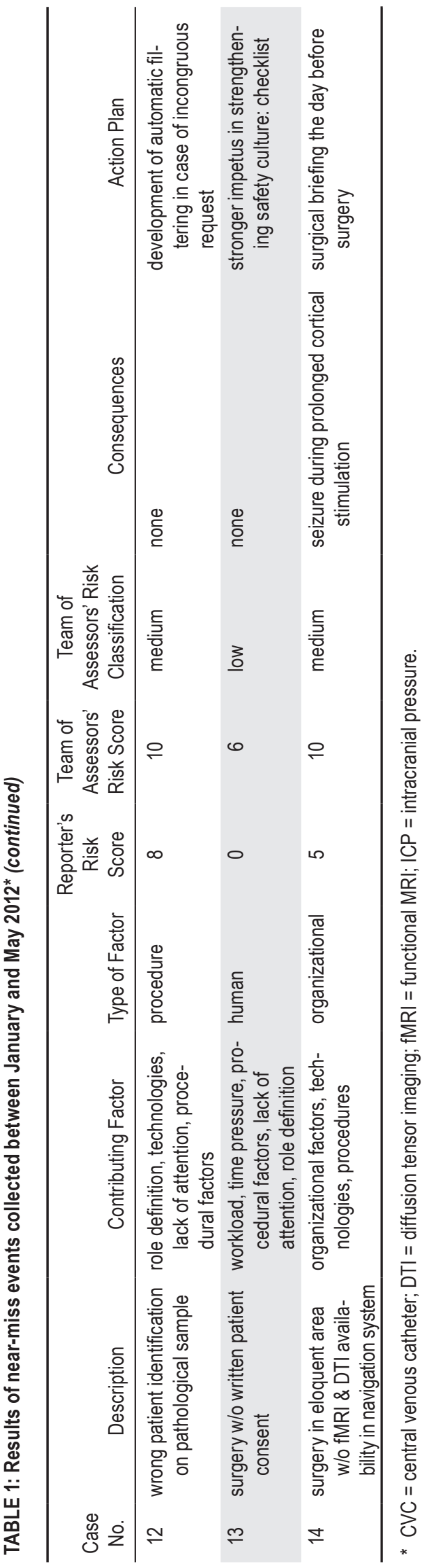

conditions, and planning; and 4) procedural factors: 1 of 14 cases-in particular, checklist effectiveness.

As far as risk stratification was concerned, 2 nearmiss events were classified by the team of assessors in the low-risk area, 7 in the middle-risk area, and 5 in the significant-risk area (Table 1).

\section{Discussion}

Incident reporting systems have significantly contributed to aviation safety and have also been found to positively influence medical care-related morbidity and mortality. Reporting systems have been implemented in several industrialized nations, such as the US, ${ }^{12,25,28}$ Germany, $8,15,18,20$ and Switzerland, which also introduced a critical national-incident reporting system., ${ }^{4,11,13}$ Safety culture first developed in anesthesiolog $\mathrm{y}^{5}$ and intensive care medicine, , $10,15,19,24,25,28$ then in other subspecialties such as hospital pharmacy, ${ }^{11,22}$ internal medicine, ${ }^{3,18}$ psychiatry, ${ }^{27}$ obstetrics and gynecology, ${ }^{13}$ pediatrics,,${ }^{1,12}$ and ambulatory care. ${ }^{4}$ Interestingly, in this regard, surgical subspecialties are still somehow underrepresented..$^{8,20}$ In performing a MEDLINE search with the terms "incident reporting AND neurosurgery" we retrieved only 1 study on this topic. ${ }^{17}$

There are difficulties in defining, identifying, and analyzing errors and responsibilities within neurosurgery; in fact, inside the medical community, we are all usually rigidly focused on the responsibility of the "end-of-the-line" operator rather than on the complex network of systemic factors that surround that operator and that may greatly obstruct safety. For instance, the operating room is a complex adaptive system in which a mix of professionals (with multiple interconnected skills and abilities) must cooperate while performing demanding technical tasks using complex technologies and techniques. ${ }^{6}$ The Reason SwissCheese model (Fig. 3) and its evolution into a health care error proliferation model effectively illustrates how the complexity of such systems, when combined with human factors, can synergistically promote errors..$^{10,22}$

Although this approach is widely accepted in aviation

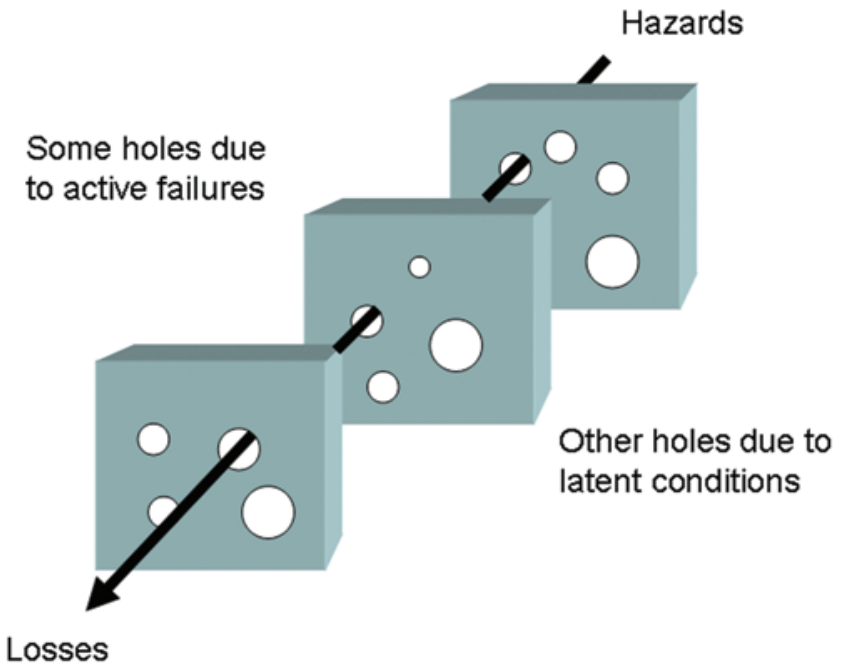

FIG. 3. Swiss-Cheese model of accident causation. Despite the presence of multiple layers of defenses, barriers, and safeguards, an error can still occur if the "holes" are all aligned. 
and some medical specialties, in surgery the question we usually ask is "Who is guilty?" rather than "Where has the system failed?" This attitude makes it difficult to foster a safety culture aimed at accident or incident prevention. Continuing to focus our attention on the end-of-the-line operator rather than trying to correct both systemic and human factors contributes to a self-perpetuating culture that has the paradoxical and unwanted effect of inviting neurosurgeons to repeat their mistakes. This was shown to be true within the aviation context, in which a "factor systems model" was instead highly effective in leading to understanding and correcting the factors that contributed to errors. ${ }^{14}$

At our institution, neurosurgeons and top managers embarked on the ISOB project, starting with error definition and describing error magnitude, for example, possible error typologies (honest mistakes vs violations or gross negligence) and the border between lapses, mistakes, and derogation. Before starting to implement a reporting system and with an institutional effort, we reinforced some concepts from the International Civil Aviation Organisation Annex 13-in particular, that "the sole objective of the investigation of an accident or incident shall be the prevention of accidents and incident" and that "it is not the purpose of this activity to apportion blame or liability." Liability in reporting is a very sensitive matter. If this problem is not handled well, the entire voluntary incident reporting system policy is jeopardized, decreasing awareness as well as motivation.

To a certain extent, near-miss or close-call events are embedded in the so-called potential effects area without any consequence to patients. Therefore, they should be considered outside of the sentinel events area, in which liability and possible legal prosecution become more relevant. According to the international aviation legislation (EU Regulation No. 996/2010 referred to incident/accident prevention in aviation), there is a double-track reporting system for near-miss or close-call events (whose reporting is anonymous, voluntary, and confidential) and sentinel events (whose reporting is mandatory). Moreover, in both reporting systems, negligence and voluntary violations to standardized protocols are not tolerated; however, this is a delicate balance, because stressing liability in the near-miss voluntary reporting process might significantly reduce reported events, thereby hampering the disclosure of sequences of events that might prevent more dangerous ones. Spontaneous reporting of any "unintentional error" should be encouraged.

Cooperating with aviation and flight safety experts was extremely helpful in implementing our incident reporting system. In this regard, the EU issued a specific directive in 2003 (2003/42/CE, for inconvenience reporting in civil aviation) in which the EU encouraged all members to avoid any frontline prosecution if the people involved in an incident were willing to reveal any useful information that might prevent similar episodes. This directive represented a milestone for civil aviation: increasing motivation, awareness, and consensus in achieving a robust and evolved incident reporting system.

Despite the investments made to foster a safety culture within our institution, the rate of reported incidents in our preliminary experience was relatively low (14 events in 5 months). In the literature, this rate ranges between 7 and 12 reports per month. ${ }^{2,15,19,20}$ In a study by Kantelhardt et al., ${ }^{17}$ reported incidents averaged 18 per month. We wonder if this discrepancy could be attributed to the fact that our form had to be downloaded from the Intranet, whereas the form in the Kantelhardt et al. study could also be filled out by hand in the operating room. Our data showed that in $64 \%$ of the cases, human factors played a significant role, confirming the trend from the literature in which human factors were involved in 51\%-79\% of reported incidents. ${ }^{2,7,9,26}$ In the series from Kantelhardt et al., human factors were involved in an even higher number of cases $(86 \%)$, confirming that most incidents can probably be prevented with a proactive attitude from the whole institution.

Organizational factors were also involved in a significant number of cases (21.4\% of cases). This result further reinforces the potentially important role of corrective actions and countermeasures developed through error analysis. Some comments regarding the Heinrich Pyramid Model should be made as regards the parallels between safety in aviation and that in the neurosurgery operating room. In fact, not all complex organizations are similar from a risk management standpoint. For instance, the stability of the system at the beginning of an aircraft flight is different from a neurosurgical operation. When a patient enters the operating room, he or she already presents some potentially critical situations because of the disease itself. Both the Reason and the Heinrich models seem to highlight the concept that usually the initial working condition in a complex organization is quite stable and that only at a later stage, in due course, criticalities might occur. Therefore, it is possible to infer that the risk of accidents in a surgical operating room must be handled at the same level of incident risk precursors without waiting for the "weak signals" to increase in frequency to anticipate the forthcoming real accident.

Neurosurgery might be an exception in which variability is much higher from the very beginning of an operation. This difference might prompt us to adopt a less conservative approach along with a traditional incident reporting system, one in which risks are investigated not only for their potential harm to patients. In such a context we also need to define our investigation framework in case we want to quickly investigate a real accident or sentinel event (that is, aviation style mortality and morbidity analysis?). First of all, it is important to set up an investigation team composed of the most experienced surgeons and representatives from all staffing constituent groups to make error analysis more accurate and faithful. It would also be important for the team to be independent, directly reporting their conclusions and suggestions for improvements to the risk manager. All team members should act within a well-defined confidentiality policy that should be double-checked and supported by a lawyer or legal expert. We are aware of the potential weakness of the whole aviation-style incident reporting apparatus that we implemented. The system surely requires validation with a higher number of analyzed cases, possibly in a multicenter or multiregion study. 


\section{P. Ferroli et al.}

\section{Conclusions}

Implementing an incident reporting system within a neurosurgery department is a complex task that should involve the entire institution, from top management to all employees. Introducing a paradigm shift away from blaming frontline operators toward an errare-humanum-est way of thinking takes time and requires a strong commitment at every institutional level. The experience and contribution from aviation safety management can be highly instrumental in fostering such a transition. Analyzing how errors happen within a specific context and what range of factors were involved in the error helps in the development of specific counter strategies to these honest mistakes (not considering intentional ones). This analysis also reinforces those barriers that will prevent most mistakes from happening again within the same system.

\section{Disclosure}

The authors report no conflict of interest concerning the materials or methods used in this study or the findings specified in this paper. Capt. Alfonso Piro is a commercial civil aviation pilot instructor and human factor consultant member of the ISOB DgSky Team. Capt. Maurizio Scholtze is an aviation safety management consultant member of the ISOB DgSky Team.

Author contributions to the study and manuscript preparation include the following. Conception and design: Ferroli. Acquisition of data: Castiglione, Broggi. Analysis and interpretation of data: Caldiroli, Perin. Drafting the article: Ferroli, Acerbi, Scholtze, Piro. Critically revising the article: Ferroli, Schiariti. Reviewed submitted version of manuscript: all authors. Study supervision: DiMeco.

\section{Acknowledgment}

The authors thank Mrs. Antonietta Dessi for invaluable help in data collection.

\section{References}

1. Ahluwalia J, Marriott L: Critical incident reporting systems. Semin Fetal Neonatal Med 10:31-37, 2005

2. Bartolomé Ruibal A, Díaz-Cañabate JI, Santa-Ursula Tolosa JA, Marzal Baró JM, González Arévalo A, García Valle del Manzano S, et al: [Application of a critical incident reporting and analysis system in an anesthesiology department.] Rev Esp Anestesiol Reanim 53:471-478, 2006 (Span)

3. Bowman L, Carlstedt BC, Black CD: Incidence of adverse drug reactions in adult medical inpatients. Can J Hosp Pharm 47:209-216, 1994

4. Brun A: [Preliminary results of an anonymous internet-based reporting system for critical incidents in ambulatory primary care.] Ther Umsch 62:175-178, 2005 (Ger)

5. Choy YC: Critical incident monitoring in anaesthesia. Med J Malaysia 61:577-585, 2006

6. Cilliers P: Complexity and Postmodernism: Understanding Complex Systems. New York: Routledge, 1998

7. Currie M, Mackay P, Morgan C, Runciman WB, Russell WJ, Sellen A, et al: The Australian Incident Monitoring Study. The "wrong drug" problem in anaesthesia: an analysis of 2000 incident reports. Anaesth Intensive Care 21:596-601, 1993

8. Domínguez Fernández E, Kolios G, Schlosser K, Wissner W, Rothmund M: [Introduction of a critical incident reporting system in a surgical university clinic. What can be achieved in a short term?] Dtsch Med Wochenschr 133:1229-1234, 2008 (Ger)
9. Fox MA, Webb RK, Singleton R, Ludbrook G, Runciman WB: The Australian Incident Monitoring Study. Problems with regional anaesthesia: an analysis of 2000 incident reports. Anaesth Intensive Care 21:646-649, 1993

10. Freestone L, Bolsin SN, Colson M, Patrick A, Creati B: Voluntary incident reporting by anaesthetic trainees in an Australian hospital. Int J Qual Health Care 18:452-457, 2006

11. Frey B, Buettiker V, Hug MI, Waldvogel K, Gessler P, Ghelfi $\mathrm{D}$, et al: Does critical incident reporting contribute to medication error prevention? Eur J Pediatr 161:594-599, 2002

12. Grant MJ, Donaldson AE, Larsen GY: The safety culture in a children's hospital. J Nurs Care Qual 21:223-229, 2006

13. Haller U, Welti S, Haenggi D, Fink D: [From the concept of guilt to the value-free notification of errors in medicine. Risks, errors and patient safety.] Gynakol Geburtshilfliche Rundsch 45:147-160, 2005 (Ger)

14. Heinrich HW: Industrial Accident Prevention: A Scientific Approach. New York: McGraw-Hill Book Company, 1931

15. Hübler M, Möllemann A, Eberlein-Gonska M, Regner M, Koch T: [Anonymous critical incident reporting system in anaesthesiology. Results after 18 months.] Anaesthesist 55:133141, 2006 (Ger)

16. International Civil Aviation Organization: Safety Management Manual (SMM): Doc 9859, AN/474, ed 2. ICAO, 2009 (http://www.icao.int/safety/ism/Guidance\%20Materials/DOC _9859_FULL_EN.pdf) [Accessed September 27, 2012]

17. Kantelhardt P, Müller M, Giese A, Rohde V, Kantelhardt SR: Implementation of a critical incident reporting system in a neurosurgical department. Cent Eur Neurosurg 72:15-21, 2011

18. Köbberling J: [The critical incident reporting system (CIRS) as a measure to improve quality in medicine.] Med Klin (Munich) 100:143-148, 2005 (Ger)

19. Madzimbamuto FD, Chiware R: A critical incident reporting system in anaesthesia. Cent Afr J Med 47:243-247, 2001

20. Missbach-Kroll A, Nussbaumer P, Kuenz M, Sommer C, Furrer M: [First experience with a critical incident reporting system in surgery.] Chirurg 76:868-875, 2005 (Ger)

21. Molloy GJ, O'Boyle CA: The SHEL model: a useful tool for analyzing and teaching the contribution of Human Factors to medical error. Acad Med 80:152-155, 2005

22. Reason JT: Human error: models and management. BMJ 320: 768-770, 2000

23. Reason JT: Managing the Risks of Organizational Accidents. Aldershot, Hampshire, UK: Ashgate, 1997

24. Reason JT, Carthey J, de Leval MR: Diagnosing "vulnerable system syndrome": an essential prerequisite to effective risk management. Qual Health Care 10 (Suppl 2):ii21-ii25, 2001

25. Vogus TJ, Sutcliffe KM: The impact of safety organizing, trusted leadership, and care pathways on reported medication errors in hospital nursing units. Med Care 45:997-1002, 2007

26. Webb RK, Currie M, Morgan CA, Williamson JA, Mackay P, Russell WJ, et al: The Australian Incident Monitoring Study: an analysis of 2000 incident reports. Anaesth Intensive Care 21:520-528, 1993

27. Wright $\mathbf{M}$, Parker G: Incident monitoring in psychiatry. J Qual Clin Pract 18:249-261, 1998

28. Wu AW, Pronovost P, Morlock L: ICU incident reporting systems. J Crit Care 17:86-94, 2002

Manuscript submitted July 15, 2012.

Accepted September 20, 2012.

Please include this information when citing this paper: DOI: 10.3171/2012.9.FOCUS12252.

Address correspondence to: Paolo Ferroli, M.D., Department of Neurosurgery, Fondazione Istituto Neurologico Carlo Besta, Via Celoria 11, 20133 Milano, Italy. email: pferroli@ istituto-besta.it. 